\title{
Controversial TV show on root canal treatment pulled
}

A controversial television documentary on the issue of root canal treatment has been removed from streaming service Netflix.

The programme called Root Cause was a feature length documentary film containing opinions from various doctors and dentists from around the world about the health effects of the root canal procedure.

It claimed that the cause of a person's fatigue, anxiety and depression was root canal treatment and also that such treatment could be linked to cancer, heart conditions and other serious illnesses due to infections inside root canals or empty spaces left behind after wisdom tooth extraction.

However, it prompted various dental organisations such as the American Dental Association to write to Netflix and other media

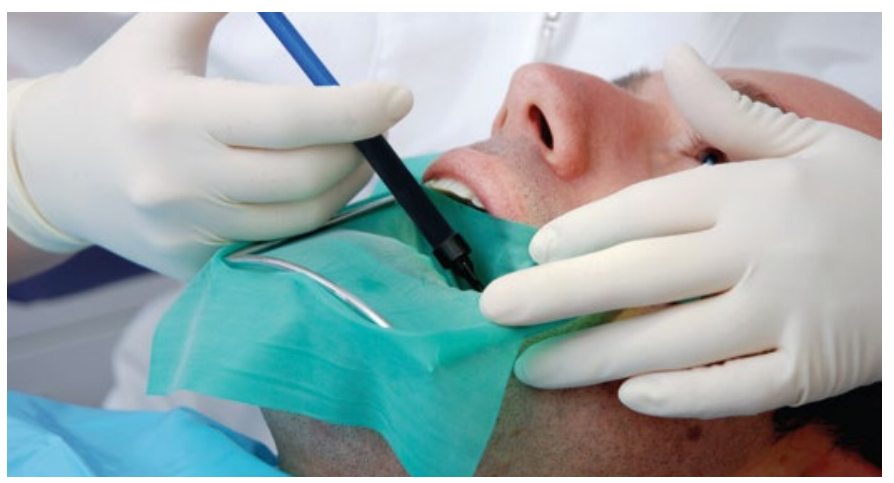

services showing the film to warn that it could harm the public by propagating long-disproven claims. Dental organisations also issued member-wide alerts about the film.

In addition, the BDA sent an email to members in which it warned the film was 'propagating misinformation about systemic health problems supposedly caused by root canal treatment.

The message said: 'These spurious claims have been countered by decades of peer-reviewed scientific evidence since they were originally made in the 1920s. We do not wish to direct public or media attention to the film by making a statement at this stage.'

Nevertheless the BDA advised its members that if they encountered any concerns from patients, they could refer them to a patient leaflet ${ }^{1}$ and inform them that:

- Root canal treatment allows an infected tooth to be saved

- The procedure removes the bacteria and prevented re-infection

- More than 500,000 endodontic treatments, including root canals, are performed safely and effectively in the UK every year

- There is no valid scientific evidence to indicate a link between root canal treatment and any other disease, including cancer.

\section{Reference}

1. BDA. Patient Leaflets - Endodontic (root canal) treatment 2018. Available at http://bda-mail.org/FS/2323/Documents/Research\%20and\%20Policy/PL17\%20-\%20 ROOT\%20CANAL\%20TREATMENT\%20WEB.PDF (accessed March 2019).

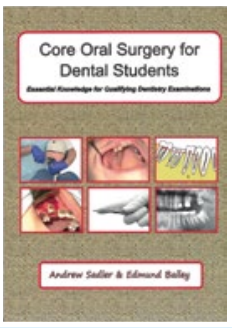

\section{CORE ORAL SURGERY FOR DENTAL STUDENTS: ESSENTIAL KNOWLEDGE FOR QUALIFYING DENTAL EXAMINATIONS}

Andrew Sadler and Edmund Bailey;

2018; Sorejaw; price £13.65; pp. 122;

ISBN: 9780956937797

This book, written by Andrew Sadler and Edmund Bailey, provides a succinct and engaging source of information concerning oral surgery. The authors' knowledge and considerable experience are apparent in the text, while being supplemented by relevant radiographs, illustrations and high-resolution clinical photographs. The resulting material is easy to read and serves as a handy learning resource and aide-memoire for the dental student or current practitioner.

The book is organised into 24 short chapters, each based around a narrow topic in oral surgery. Topics covered include history-taking and examination, surgical armamentarium, assessment for extraction, simple and surgical exodontia, complications, spreading infection, benign and malignant lesions of the soft and hard tissues, jaw fractures and biopsy, to name a few.

The use of boxes and tables contribute to a reader-friendly method of delivering select information with fine detail, as well as regularly placed red 'examination tips' boxes that are indispensable to the aspiring dental student. Towards the end of the book, a section dedicated to undergraduate examination tips is included, which contains common methods of assessment alongside example questions and scenarios. The final appendix details a list of guidelines and protocols written by professional bodies and societies for use in the management of oral surgery-related conditions. This is a particularly useful addition for students looking for up-to-date, evidence-based guidance in preparation for examinations, vivas and treating patients.

The book focuses more on the practical aspects of oral surgery rather than the minutiae of theory. If you are trying to find out the histological appearance of a central giant cell granuloma, you won't find it here. Instead, this text provides a no nonsense guide to everyday oral surgery, with the rarer conditions getting a brief mention.

The chapters on surgical extraction and impacted third molars, in particular, are valuable, as they demystify a commonly occurring technical challenge for the undergraduate dental student. The guide to surgical removal of third molars was neatly finished with a pragmatic discussion of the NICE guidance relating to their extraction, encompassing the potential pitfalls, real life application and future changes to the guidelines.

This book is highly recommended to any undergraduate dental student looking for an engaging and realistic guide to oral surgery. With helpful examination tips and real world advice, this book is certainly beneficial for the student looking to increase their confidence and knowledge in oral surgery.

By Ishita Thakrar and Indriyesha Puri 